\title{
Brachial Plexopathy
}

National Cancer Institute

\section{Source}

National Cancer Institute. Brachial Plexopathy. NCI Thesaurus. Code C27194.

A brachial plexus disorder characterized by regional paresthesia, pain and muscle weakness, and limited movement in the arm or hand. 